\title{
Oxidized CaMKII causes cardiac sinus node dysfunction in mice
}

\author{
Paari Dominic Swaminathan,, ${ }^{1}$ Anil Purohit, ${ }^{1}$ Siddarth Soni, ${ }^{2}$ Niels Voigt, ${ }^{3}$ Madhu V. Singh, ${ }^{1}$ \\ Alexey V. Glukhov, ${ }^{4}$ Zhan Gao, ${ }^{1}$ B. Julie He, ${ }^{1}$ Elizabeth D. Luczak, ${ }^{1}$ Mei-ling A. Joiner, ${ }^{1}$ \\ William Kutschke, ${ }^{1}$ Jinying Yang, ${ }^{1} \mathrm{~J}$. Kevin Donahue, ${ }^{5}$ Robert M. Weiss, ${ }^{1}$ Isabella M. Grumbach, ${ }^{1}$ \\ Masahiro Ogawa, ${ }^{6,7}$ Peng-Sheng Chen, ${ }^{6}$ Igor Efimov, ${ }^{4}$ Dobromir Dobrev, ${ }^{3}$ Peter J. Mohler, ${ }^{1,8}$ \\ Thomas J. Hund, ${ }^{1,9}$ and Mark E. Anderson ${ }^{1,8}$
}

\begin{abstract}
1Division of Cardiovascular Medicine, Department of Internal Medicine, Carver College of Medicine, University of lowa, lowa City, Iowa, USA.
2Division of Heart and Lungs, Department of Medical Physiology, University Medical Centre Utrecht, Utrecht, The Netherlands.

3Department of Pharmacology and Toxicology, Dresden University of Technology, Dresden, Germany, and Division of Experimental Cardiology,

Medical Faculty Mannheim, University of Heidelberg, Mannheim, Germany. ${ }^{4}$ Department of Biomedical Engineering, Washington University,

St. Louis, Missouri, USA. ${ }^{5}$ MetroHealth Medical Center, Cleveland, Ohio, USA. ${ }^{6}$ Krannert Institute of Cardiology, Indiana University School of Medicine, Indianapolis, Indiana, USA. ${ }^{7}$ Department of Cardiology, Fukuoka University School of Medicine, Fukuoka, Japan.

${ }^{8}$ Department of Molecular Physiology and Biophysics and ${ }^{9}$ Department of Biomedical Engineering, University of lowa, lowa City, Iowa, USA.
\end{abstract}

\begin{abstract}
Sinus node dysfunction (SND) is a major public health problem that is associated with sudden cardiac death and requires surgical implantation of artificial pacemakers. However, little is known about the molecular and cellular mechanisms that cause SND. Most SND occurs in the setting of heart failure and hypertension, conditions that are marked by elevated circulating angiotensin II (Ang II) and increased oxidant stress. Here, we show that oxidized calmodulin kinase II (ox-CaMKII) is a biomarker for SND in patients and dogs and a disease determinant in mice. In wild-type mice, Ang II infusion caused sinoatrial nodal (SAN) cell oxidation by activating NADPH oxidase, leading to increased ox-CaMKII, SAN cell apoptosis, and SND. $47^{-}-$mice lacking functional NADPH oxidase and mice with myocardial or SAN-targeted CaMKII inhibition were highly resistant to SAN apoptosis and SND, suggesting that ox-CaMKII-triggered SAN cell death contributed to SND. We developed a computational model of the sinoatrial node that showed that a loss of SAN cells below a critical threshold caused SND by preventing normal impulse formation and propagation. These data provide novel molecular and mechanistic information to understand SND and suggest that targeted CaMKII inhibition may be useful for preventing SND in high-risk patients.
\end{abstract}

\section{Introduction}

Each normal heart beat is initiated as an electrical impulse from a small number of highly specialized sinoatrial node (SAN) pacemaker cells that reside in the lateral right atrium. There is now general agreement that physiological SAN function requires a pacemaker current $\left(I_{f}\right)(1)$ and spontaneous release of sarcoplasmic reticulum (SR) intracellular $\mathrm{Ca}^{2+}$ that triggers depolarizing current through the $\mathrm{Na}^{+} / \mathrm{Ca}^{2+}$ exchanger $\left(I_{N C X}\right)(2,3)$. The multifunctional $\mathrm{Ca}^{2+} /$ calmodulin-dependent protein kinase II (CaMKII) is essential for increasing $\mathrm{SR} \mathrm{Ca}^{2+}$ release in SAN cells in response to stress to cause physiological "fight-or-flight" heart rate (HR) increases (4). Although the physiological basis for SAN behavior is increasingly understood, very little is known about SAN disease. Severe SAN dysfunction (SND) is marked by irregular prolonged pauses between heart beats, pathologically slow HRs at rest, and inadequate activity-related increases in HR. At present, surgical implantation of permanent pacemakers is required for treatment of SND and costs \$2 billion annually in the United States (5). SND commonly occurs in the setting of heart failure and hypertension (6-8), conditions characterized by excessive activation of renin-Ang II signaling (9) and elevated levels of ROS (10). Ang II increases ROS in ventricular myocardium by stimulating NADPH oxidase to cause activation of CaMKII (ox-CaMKII) by oxidation of Met281/282 in the CaMKII regulatory domain (11).

Conflict of interest: Mark E. Anderson is a cofounder of Allosteros Therapeutics, a biotech company developing therapeutic enzyme inhibitors.

Citation for this article: J Clin Invest. 2011;121(8):3277-3288. doi:10.1172/JCI57833.
CaMKII is initially activated by binding to calcified calmodulin, but Met oxidation traps CaMKII in a persistently active conformation even in the absence of $\mathrm{Ca}^{2+}$-bound calmodulin. Ang II-induced oxCaMKII promotes myocardial dysfunction and heart failure in part by increasing apoptosis in ventricular myocytes (11). Based on this information, we reasoned that the relatively smaller number of cells in the SAN compared with the ventricular myocardium would make physiological SAN function particularly vulnerable to SAN cell death. Here we asked whether excessive ox-CaMKII could be a molecular determinant of SND by promoting SAN cell apoptosis.

We found that right atrial tissue from patients with heart failure who required artificial pacemakers for SND had more ox-CaMKII compared with patients with heart failure alone and patients without heart failure or SND. Similarly, dogs with pacing-induced heart failure and SND also had elevated right atrial ox-CaMKII compared with non-SND controls. In order to test whether elevated ox-CaMKII could cause SND, we infused mice with Ang II. Ang II infusion for 3 weeks caused increased SAN ox-CaMKII, SAN cell apoptosis, fibrosis, slowed atrial impulse conduction velocity, and SND. We prevented Ang II-triggered SND by transgenic myocardial and SAN cell expression of a synthetic CaMKII inhibitory peptide (AC3-I) (12) and by SAN-targeted gene therapy providing ectopic SAN expression of a CaMKII inhibitory peptide, CaMKIIN (13), that is endogenous to neurons but absent in heart. Neither transgenic nor gene-targeting approaches to SAN CaMKII inhibition affected the hypertensive response to Ang II, nor did they abrogate the increased SAN ROS due to Ang II infusion, indicating 

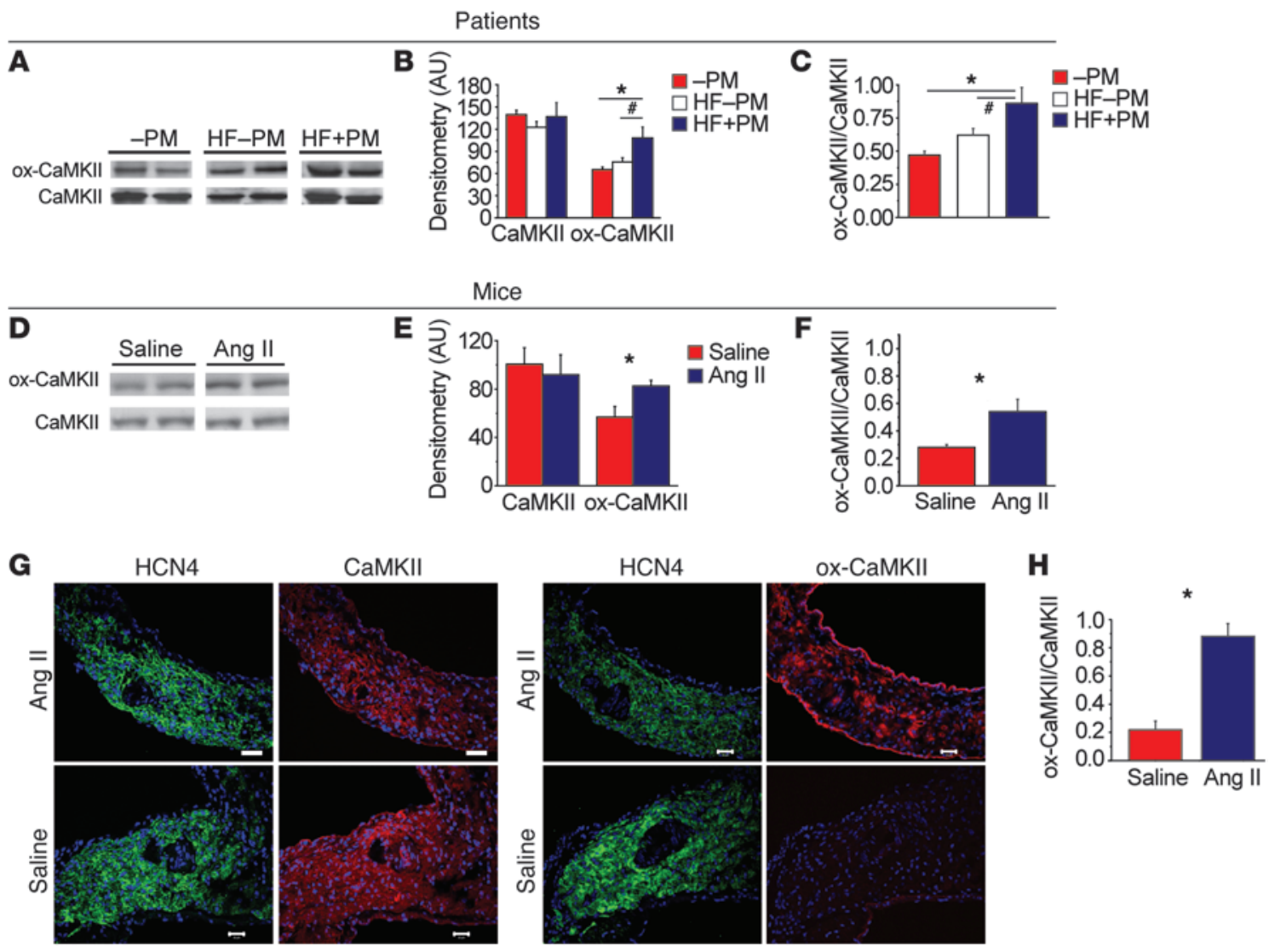

\section{Figure 1}

ox-CaMKII is increased in heart failure patients and in Ang II-infused mice. (A) Representative immunoblots showing ox-CaMKII and total CaM$\mathrm{KIl}$ from right atrial tissue obtained from heart failure patients with SND requiring pacemaker (HF+PM) implantation, heart failure patients without SND (HF-PM), and controls (-PM). The lanes were run on the same gel but were noncontiguous. (B) Summary data for ox-CaMKII and total CaMKII in HF+PM $(n=5)$, HF-PM $(n=6)$, and -PM $(n=10)$ patients $\left({ }^{*} P<0.05\right.$, $\left.{ }^{*} P<0.01\right)$. (C) Increased ox-CaMKII normalized to total CaMKII from patient samples shown in $\mathbf{B}\left({ }^{\star} P<0.05\right.$ and $\left.{ }^{\#} P<0.01\right)$. (D) Representative immunoblots showing ox-CaMKII and total CaMKII from right atrial tissue obtained from WT mice infused with Ang II or saline for 3 weeks. The lanes were run on the same gel but were noncontiguous. (E) Summary data for ox-CaMKII and total CaMKII in Ang II- $(n=6)$ and saline-infused $(n=6)$ mice $\left({ }^{\star} P=0.03\right)$. (F) Increased ox-CaMKII normalized to total CaMKII in mouse samples shown in $\mathbf{E}\left({ }^{\star} P=0.02\right)$. (G) Representative immunofluorescence images showing that 3 weeks of Ang II infusion increases SAN ox-CaMKII. SAN area is identified by HCN4 immunostaining (green). Scale bars: $50 \mu m$. (H) Summary data showing increased ox-CaMKII normalized to total CaMKII in SAN tissue from mice treated with 3 weeks of Ang II or saline infusion $\left(n=4 /\right.$ group, $\left.{ }^{*} P<0.01\right)$.

that CaMKII was a critical downstream signal for the pathological actions of ROS on SAN. The increase in SAN ox-CaMKII by Ang II required activation of NADPH oxidase, because it was absent in $p 47^{-1-}$ mice lacking functional NADPH oxidase. We developed a structural and computational model of the SAN that revealed a quantitative mechanism to explain how Ang II-induced SAN cell apoptosis resulted in SND by reducing SAN cell number and increasing electrotonic loading of surviving SAN cells to cause loss of high-fidelity impulse formation and propagation. Our findings provide insights into how excessive activation of CaMKII in SAN cells causes SND and identify what we believe to be a novel approach to reducing SND by CaMKII inhibition.

\section{Results}

ox-CaMKII is increased in SND. Increasing evidence supports the view that physiological activation of CaMKII enables core myocardial activities, such as excitation-contraction coupling in ventricular myocardium (14) and cardiac pacing in SAN cells (4), while excessive activation of CaMKII by ROS contributes to myocardial dysfunction and heart failure (11). As a first step in examining the potential relationship of ox-CaMKII to SND, we asked whether oxCaMKII is elevated in patients with severe SND, as evidenced by a requirement for a surgically implanted permanent pacemaker. Our biobank included heart failure patients with SND, heart failure patients without SND, and control patients without heart failure or SND (Supplemental Table 1; supplemental material available online with this article; doi:10.1172/JCI57833DS1). We measured ox-CaMKII, using a validated antiserum against oxidized Met281/282 in the CaMKII regulatory domain (11), in right atrial samples. The differences between the 3 groups in absolute level of ox-CaMKII and ratio of ox-CaMKII to total CaMKII were significant $(P=0.003$ [ANOVA] for ox-CaMKII and $P=0.001$ for the ratio), but the only post hoc (Tukey's test) differences that reached significance were between controls and heart failure patients with SND and between heart failure patients with and without SND (Figure 1, A-C). The LV ejection fraction (LVEF) was similar in heart 
A In vivo
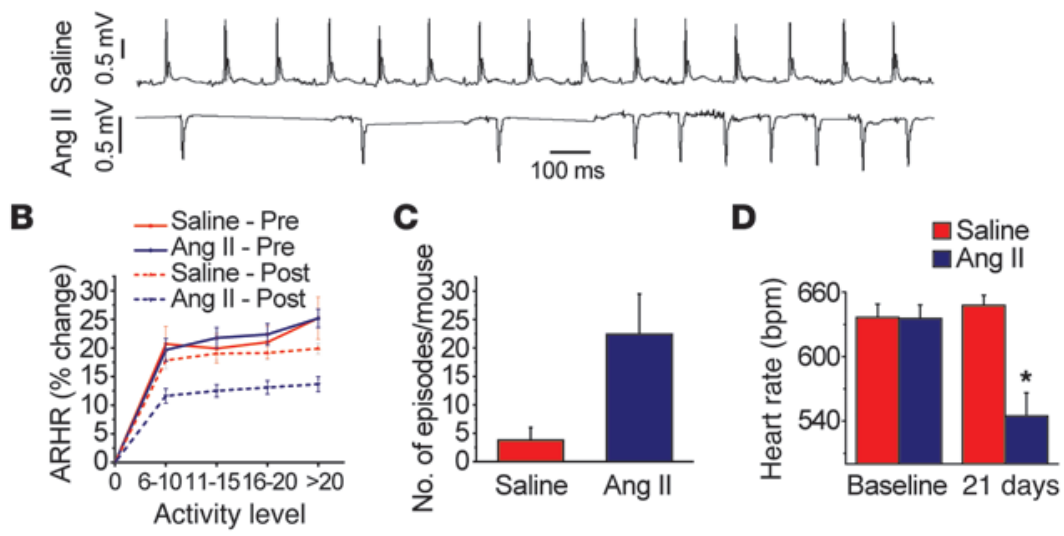

E Ex vivo
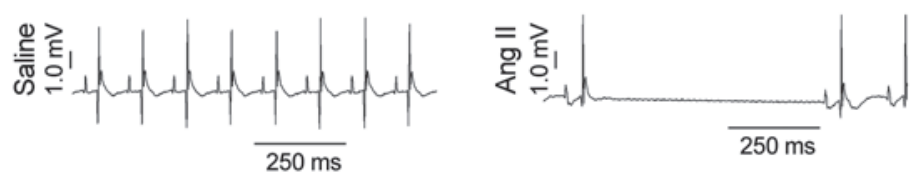

F $\square$ Sinus pause/exit block

$\square$ No sinus pause/exit block

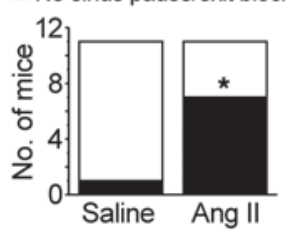

G

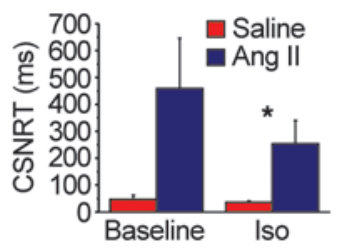

\section{Figure 2}

Ang II infusion causes SND. (A-D) In vivo data from unanesthetized and unrestrained ECG-telemetered mice. (E-H) Ex vivo data from Langendorff-perfused mouse hearts. (A) A representative ECG recording of spontaneous bradycardia in an Ang II-infused mouse and a normal ECG recording in a saline-infused mouse. (B) Diminished spontaneous activity-responsive HR (ARHR) $(P<0.01)$ in mice after 3 weeks of Ang II infusion compared with other groups (5 mice/group). Pre, prior to miniosmotic pump insertion; Post, 3 weeks after infusion. (C) Summary data showing a trend $(P=0.06$, $n=4-5$ /group) toward reduced episodes where HR was less than 200 beats/min in Ang II-compared with saline-infused mice. (D) Ang II infusion for 3 weeks reduced resting in vivo HR, compared with 3 weeks of saline infusion ( $n=25$ /group, $P<0.001)$. (E) Representative ECG recordings from Langendorff-perfused hearts isolated from mice infused with Ang II or saline for 3 weeks. (F) Summary data showing that Ang II-infused mice have more sinus pauses than saline-infused mice $\left({ }^{*} P=0.023\right.$, $n=11 /$ group). (G) Hearts isolated from Ang IIinfused mice have prolonged CSNRT compared with saline-infused controls $\left({ }^{*} P=0.04, n=10\right)$. Iso, isoproterenol. failure patients with and without SND (Supplemental Table 1). Dogs with pacing-induced heart failure and SND also had significantly $(P=0.02)$ increased ox-CaMKII in right atrial tissue, compared with controls without pacing-induced heart failure or SND (Supplemental Figure 1, A-C). Thus, we observed increased ox-CaMKII in right atrial tissue from both patients and dogs with SND and heart failure.

Based on this potential link between Ang II and ox-CaMKII in SND, we developed a mouse model of SND. Heart failure patients develop an approximately 6-fold increase in plasma Ang II levels compared with non-hypertensive healthy controls $(15,16)$. We infused WT mice with Ang II $(2,000 \mathrm{ng} / \mathrm{kg} / \mathrm{min})$ or an equal volume of saline for 3 weeks to produce a 4 -fold increase in plasma Ang II compared with that in control mice infused with saline for the same period of time (Supplemental Figure 2). Thus, the elevation in Ang II levels in our mice over baseline approximated the fold increases in plasma Ang II observed in patients with heart failure at increased risk for SND. Ang II infusion significantly $(P=0.02)$ enhanced right atrial (Figure $1, \mathrm{D}$ and $\mathrm{F})$ and SAN ox-CaMKII $(P<0.01$; Figure $1, \mathrm{G}$ and $\mathrm{H})$ compared with salineinfused mice, indicating that the right atrial and SAN tissues exhibited coordinate increases in ROS in response to Ang II. In contrast to the changes in ox-CaMKII, total right atrial CaMKII expression was equivalent in patients and dogs with or without SND (Figure 1, A and B, and Supplemental Figure 1, A and B) and in Ang II- and saline-infused mice (Figure 1, D and E). The intensity of HCN4 staining appeared decreased in the SAN of Ang IIinfused compared with saline infused mice, potentially consistent with a decrease in SAN cell density and/or an increase in connec- tive tissue, features known to occur in aging-induced SND (17). Taken together, these findings suggested that elevated right atrial ox-CaMKII is a biomarker for SND in humans and dogs and that Ang II can enhance right atrial and SAN ox-CaMKII, similar to findings in ventricular myocardium (11).

Ang II infusion causes SND. In order to determine whether our model of Ang II infusion promoted SND, we measured HR in unrestrained and unanesthetized mice implanted with ECG and activity-sensing telemeters. Ang II-infused mice exhibited frequent sinus pauses (Figure 2A), similar to patients with SND (8). SND patients may show diminished activity-evoked increases in HR, so we next examined the relationship between activity and HR in our telemetered mice (Figure 2B). Ang II infusion significantly $(P<0.01)$ reduced the maximum $\mathrm{HR}$ response to spontaneous activity (by $45 \% \pm 5 \%$ ), whereas mice with saline infusion showed no significant change in HR response to spontaneous activity compared with mice prior to implantation of osmotic minipumps (Figure 2B). The saline and Ang II groups remained equally active at the end of 3 weeks (Supplemental Figure 3), suggesting that Ang II induced SND without causing heart failure or severe systemic illness. Echocardiographic studies showed that Ang II-infused mice had increased LV hypertrophy $(P=0.02)$ without reduction in LVEF (Supplemental Table 2). The Ang IIinfused mice showed a trend $(P=0.06)$ toward more frequent episodes of severe HR slowing (Figure 2C) and a significant $(P<0.001)$ decrease in resting HR, whereas resting HR was unchanged after 3 weeks of saline infusion (Figure 2D). Collectively, these data showed that Ang II infusion results in elevated SAN ox-CaMKII (Figure 1, G and H) and SND (Figure 2, A-D). 


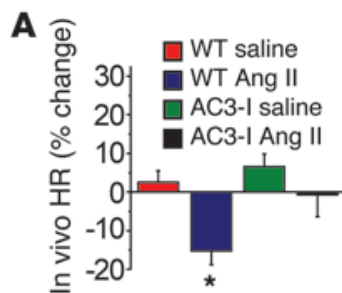

D
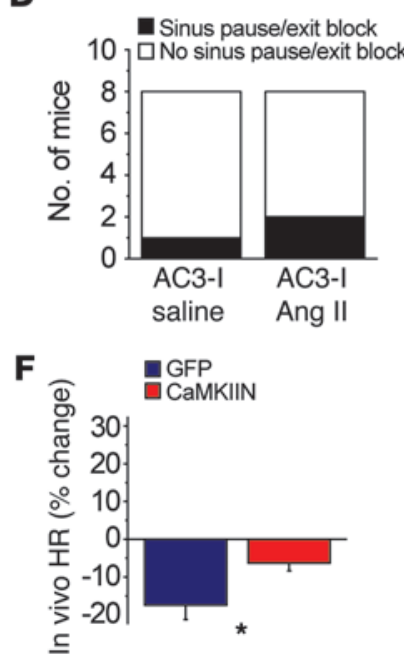

B

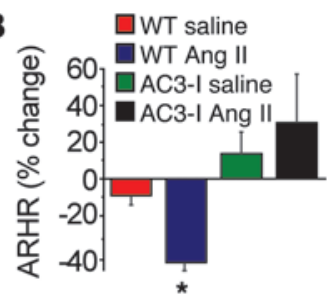

E

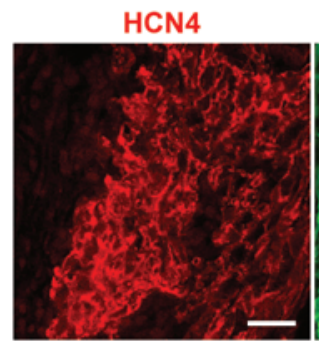

G

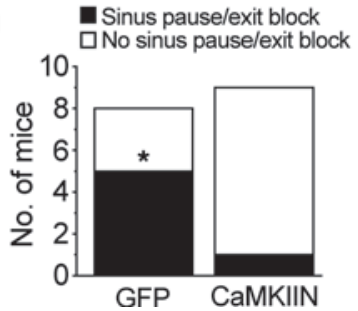

C

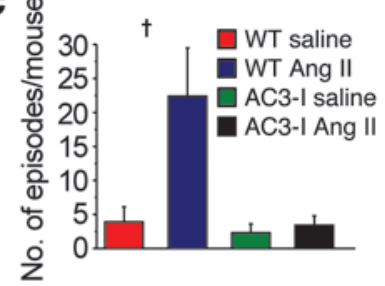

GFP

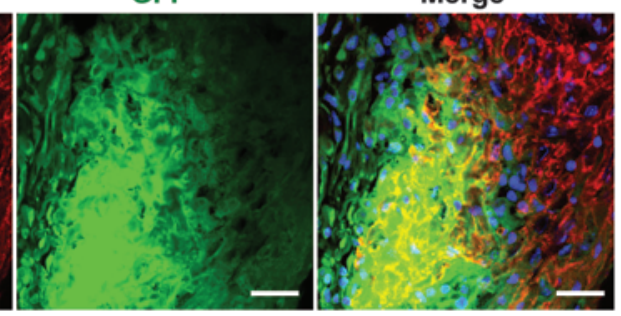

H

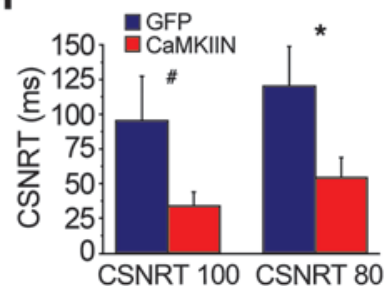

Figure 3

Myocardial and SAN-targeted CaMKII inhibition protects against SND. (A) ECG-telemetered, unrestrained, and unanesthetized AC3-I transgenic mice ( $n=8-10 /$ group) infused with Ang II for 3 weeks have no significant decrease in resting HR or (B) reduction in ARHR or (C) increased bradycardia events. ${ }^{*} P<0.01$ compared with baseline; ${ }^{\dagger} P<0.01$ compared with all other groups. (D) Ex vivo Langendorff-perfused AC3-I hearts isolated after 3 weeks of Ang II infusion have no significant increase in sinus pauses ( $n=8 /$ group). (E) Gene painting with an adenovirus-poloxamer mixture (see Methods) allowed for SAN-targeted expression of IRES eGFP from the CaMKIIN adenovirus (scale bars: 25 um). (F) WT mice ( $n=5-6$ /group) with SAN expression of CaMKIIN and eGFP were significantly ( ${ }^{*} P=0.02$ ) resistant to reduced resting HR after 3 weeks of Ang II infusion compared with WT mice with SAN eGFP expression alone. (G) Langendorff-perfused hearts from WT mice with SAN-targeted expression of CaMKIIN and eGFP were significantly ( ${ }^{\star} P=0.049, n=5-6 /$ group) protected against Ang Il-induced increases in sinus pauses compared with WT mice expressing eGFP alone. (H) SAN-targeted CaMKIIN expression significantly protected against Ang II infusion induced CSNRT prolongation in response to $80-\mathrm{ms}\left({ }^{\star} P=0.04\right)$ and $100-\mathrm{ms}\left({ }^{\#} P=0.049\right)$ pacing cycle intervals. The same hearts were studied in $\mathbf{G}$ and $\mathbf{H}$.

In vivo $H R$ is an integrated response that reflects intrinsic SAN properties and autonomic nerve activity. In order to test whether Ang II-induced SND involved direct effects on SAN tissue, we measured HR in Langendorff-perfused hearts isolated from mice infused with Ang II or saline for 3 weeks. Significantly more hearts isolated from Ang II-infused mice $(P=0.02)$ showed pauses characteristic of SND, where the P-P wave interval was greater than or equal to twice the baseline sinus cycle length (Figure 2, E and F), compared with saline-infused control mice. We next measured sinus node recovery time corrected for HR (CSNRT), a clinical index of SAN function $(7,8,18)$, in the Langendorff-perfused hearts. There was a trend $(P=0.09)$ toward longer CSNRT in mice treated with Ang II compared with saline at baseline, while the CSNRT was significantly $(P=0.04)$ prolonged in Ang II-treated mice compared with saline-treated mice in the presence of isoproterenol (10 nM, Figure 2G). We considered the possibility that the reduced $\mathrm{HR}$ response to activity (Figure $2 \mathrm{~B}$ ) and the prolongation in CSNRT in the presence of isoproterenol (Figure 2G) in Ang IIinfused mice were due to defects in SAN cell $\beta$-adrenergic receptor $(\beta-A R)$ signaling. However, there were no differences in mRNA levels of $A d r b 1$, the gene encoding $\beta 1$-AR, in SAN tissue from saline- or Ang II-treated mice (Supplemental Figure 4A). Furthermore, activity of PKA, a critical downstream signal activated during $\beta 1$-AR agonist stimulation (19), was preserved in Ang II-treated compared with saline-treated WT mice $(P=0.9$, Supplemental Figure $4 \mathrm{~B})$ exposed to isoproterenol $(10 \mathrm{nM})$. We interpreted these findings to suggest that SND phenotypes in Ang II-infused mice were not primarily due to impaired responses of the SAN $\beta$-AR pathway. Taken together, these findings support the hypothesis that Ang II infusion resulted in SND in vivo and ex vivo, at least in part, by directly altering intrinsic SAN cell and/or tissue properties.

Myocardial CaMKII inbibition protects against Ang II-induced SND. Based on our data showing increased ox-CaMKII in patients and dogs with SND (Figure 1 and Supplemental Figure 1) and previous studies showing myopathic effects of excessive ox-CaMKII in ventricular myocardium (11), we hypothesized that ox-CaMKII is a determinant of Ang II-induced SND. To test this hypothesis, we first used transgenic mice with cardiomyocyte (12) and SAN (4) transgenic expression of AC3-I, a synthetic CaMKII inhibitory peptide. AC3-I mice have a normal resting HR (Supplemental Figure 6A) but blunted HR responses to stress, because CaMKII inhibition reduces stress-responsive $\mathrm{SR} \mathrm{Ca}^{2+}$ release that is required for 


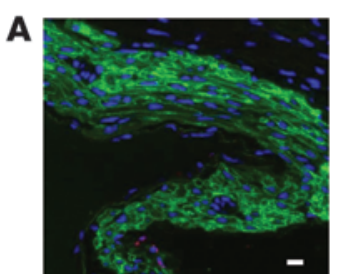

Saline

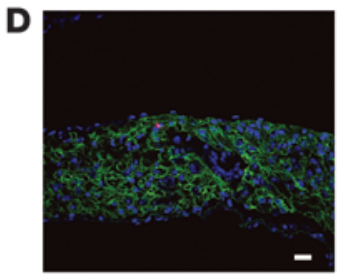

AC3-I Saline

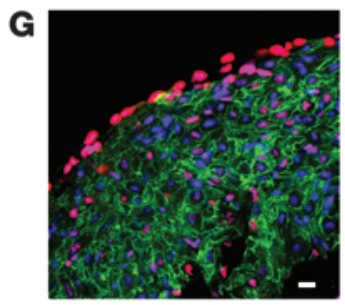

GFP

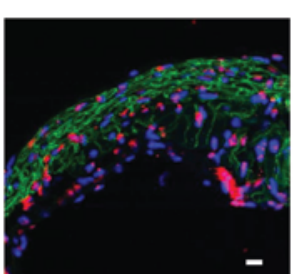

Ang II

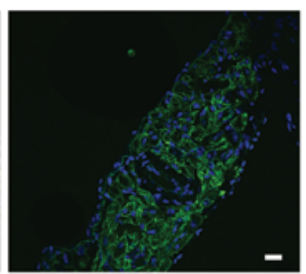

AC3-I Ang II

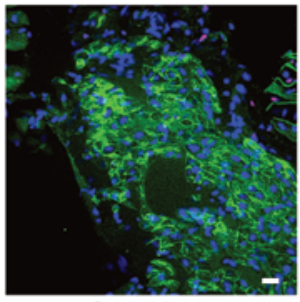

CAMKIIN
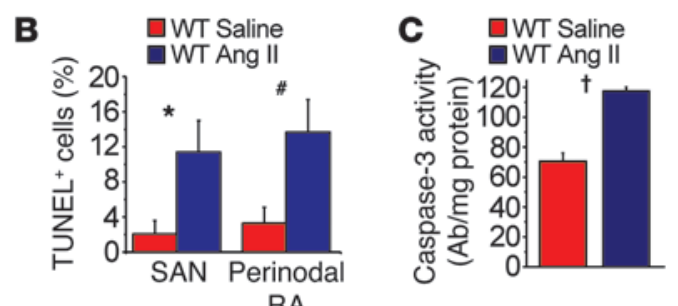

E
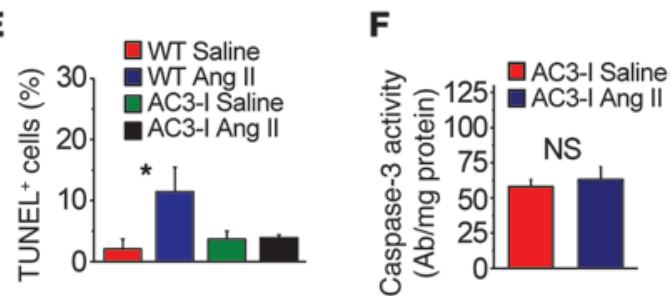

Figure 4

Ang II infusion causes SAN cell death and SND. (A) Representative SAN tissue section showing increased TUNEL staining (red) in mice infused with Ang II compared with saline-infused mice. Blue staining shows nuclei (DAPI), and green shows HCN4; scale bars: $20 \mu \mathrm{m}$ for all micrograph panels. (B) Summary data showing increased TUNEL-positive cells in the SAN (intranodal, ${ }^{*} P=0.001, n=5 /$ group) and in SAN-adjacent tissue (perinodal, ${ }^{\#} P=0.006, n=5 /$ group) and (C) activated caspase- 3 compared with saline-infused controls $\left({ }^{\dagger} P<0.001, n=7 /\right.$ group). (D) Representative SAN tissue sections showing that Ang II infusion for 3 weeks does not increase TUNEL staining in AC3-I mice. (E) Summary data showing that the AC3-I mice infused with Ang II for 3 weeks are resistant to SAN cell death $\left(P=0.89\right.$ for AC3-I Ang II versus saline and ${ }^{*} P=0.001$ for WT Ang II versus saline, $n=5 /$ group) and $(\mathbf{F})$ caspase-3 activation $(P=0.73)$ compared with saline-infused controls. (G) Representative tissue sections showing that SAN-targeted eGFP and CaMKIIN expression protects against Ang II-induced TUNEL staining compared with SAN-targeted eGFP expression alone in WT mice. (H) Summary data from studies in $\mathbf{G}$ ( ${ }^{*} P=0.001, n=6 /$ group).

physiological increases in HR (4). We infused AC3-I mice with Ang II or vehicle for 3 weeks. Ang II- and saline-infused AC3-I mice had equivalent resting HR after 3 weeks (Figure 3A), and AC3-I mice did not show reduced HR responses to spontaneous activity after Ang II compared with saline infusion (Figure 3B). In contrast, WT mice showed a significant decrease in resting (Figure $3 \mathrm{~A}$ ) and activity-responsive HR (Figure 3B) after Ang II infusion. In contrast to our observations in WT mice (Figure 2C), Ang II infusion was ineffective in increasing bradycardia episodes during spontaneous activity in AC3-I mice (Figure 3C). We measured BP to test whether the resistance of AC3-I mice to Ang II-induced SND was due to alterations in the hypertensive effects of Ang II. However, AC3-I and WT mice had normal (equivalent) hypertensive responses to Ang II (Supplemental Figure 5), indicating that CaMKII inhibition protected against SND without affecting the pressor response to Ang II. Langendorff-perfused hearts isolated from AC3-I mice infused with Ang II were no more likely to exhibit spontaneous pauses than AC3-I hearts infused with saline (Figure 3D), in contrast to our observations that WT mouse hearts infused with Ang II had increased susceptibility to spontaneous pauses (Figure 2, A, C, and D). The CSNRT was unaffected by 3 weeks of Ang II treatment in AC3-I mice and remained similar to the CSNRT measured in saline-treat- ed zWT mice (Supplemental Figure 6B). Our data so far showed that CaMKII inhibition protects against SND in vivo and ex vivo, supporting the concept that activation of the CaMKII pathway in SAN by oxidation is required for Ang II-induced SND.

We next sought to determine whether SAN-targeted CaMKII inhibition was sufficient to prevent SND, in the absence of "global" myocardial CaMKII inhibition, and potentially provide proof-of-concept evidence to support the use of CaMKII inhibitor gene therapy to prevent SND. We developed a SAN-targeted gene transfer technique to deliver the most potent and selective known biological CaMKII inhibitor, CAMKIIN, a peptide that is endogenous to neurons but is not expressed in heart (20). We fused the minigene for CaMKIIN to eGFP and incorporated the cDNA into adenovirus. The CaMKIINeGFP-encoding adenovirus or an adenovirus encoding eGFP alone was suspended in a poloxamer gel that enhances efficacy of myocardial viral infection (21). We performed SAN painting with the CaMKIIN-eGFP- or eGFP-expressing adenovirus on WT Ang II-infused mice (Figure 3E). CaMKIIN-expressing mice infused with Ang II for 3 weeks had significant $(P=0.01)$ protection from a decline in resting HR compared with Ang II-infused mice with SAN tissue expressing eGFP alone ( $6 \% \pm 2 \%$ versus $18 \% \pm 3 \%$, Figure $3 F)$. Significantly fewer Langendorff-perfused hearts isolated from mice with 

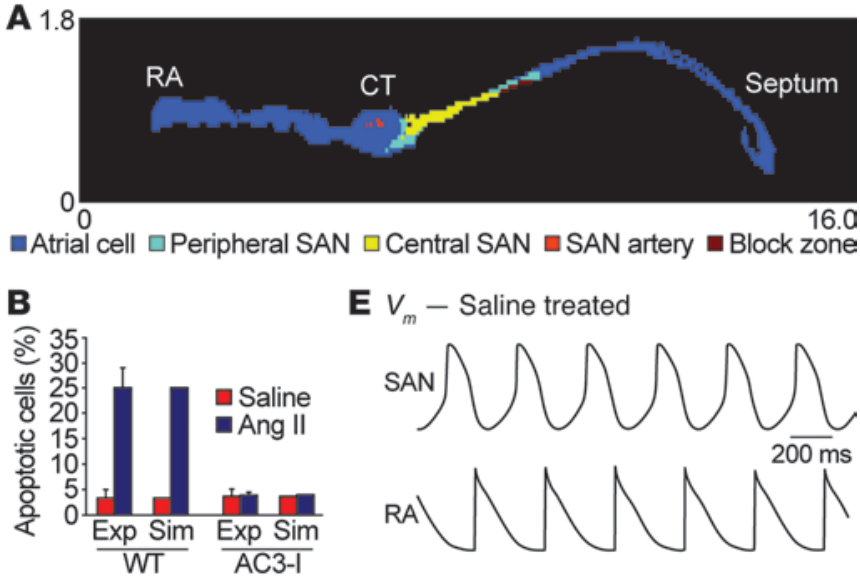

E $v_{m}-$ Saline treated
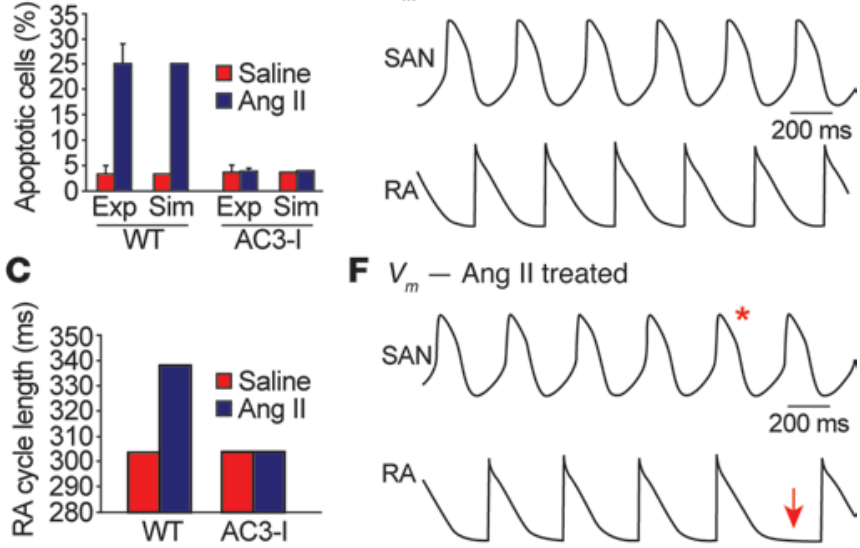

F $V_{m}-$ Ang II treated
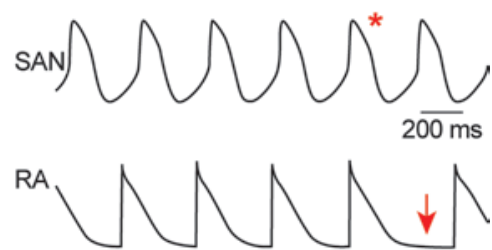

D

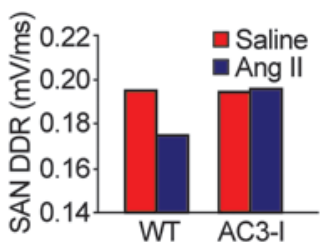

SAN CaMKIIN expression compared with eGFP alone $(P=0.049)$ showed spontaneous pauses (Figure $3 \mathrm{G}$ ). SAN painting with CaMKIIN significantly $(P=0.04)$ reduced CSNRT prolongation compared with eGFP painting (Figure $3 \mathrm{H}$ ) in mice infused with Ang II. These data show that SAN-targeted CaMKII inhibition is sufficient to protect against Ang II-induced SND and suggest that SAN CaMKII is a potential new target for preventing SND in common cardiac disease conditions, such as heart failure and hypertension.

Ang II causes SND by CaMKII-triggered SAN cell apoptosis. Our experiments to this point confirmed that the CaMKII pathway is a critical determinant of Ang II-mediated SND, but the mechanism underlying pathological actions of CaMKII on SAN tissue was unclear. Based on previous studies showing that Ang II increased ox-CaMKII leading to apoptosis in ventricular myocytes (11), we hypothesized that elevated ox-CaMKII following Ang II infusion resulted in SND, at least in part, by promoting excessive SAN cell death. We found that SAN tissue from Ang II-infused WT mice had significantly $(P=0.001)$ increased TUNEL staining (Figure 4, $A$ and $B$ ) and caspase- 3 activity $(P<0.001$, Figure $4 C)$, indicating increased cell death (11), compared with SAN tissue from salinetreated mice. In contrast, AC3-I mice were resistant to Ang IIinduced increases in TUNEL staining (Figure 4, D and E) and caspase-3 activity (Figure 4F). WT mice with CaMKIIN-expressing SAN tissue were also resistant to Ang II-induced increases in TUNEL staining (Figure 4, $\mathrm{G}$ and $\mathrm{H}$ ), compared with controls with SAN eGFP expression alone. The consistency of the findings, by two distinct methods, that CaMKII inhibition reduced SND and

\section{Figure 5}

A structurally based mathematical model of the SAN. (A) Structural model of 2-dimensional tissue slice with color-coded representation of different regions, including atrial cell, peripheral SAN, and central SAN zones. CT, crista terminalis. (B) Experimentally measured and simulated percentage of apoptotic cells in WT and AC3-I SAN following infusion with saline or Ang II. (C) Simulated activation cycle length of right atrial (RA) free wall during sinus rhythm in the WT and AC3-I tissue model following 3 weeks of infusion with saline or Ang II. Ang II infusion slows RA activation in the WT but not in the AC3-I model. Exp, experimental; Sim, simulated. (D) SAN diastolic depolarization rate (DDR) from the central SAN region in the WT and AC3-I models following saline or Ang II infusion. Ang II infusion slows SAN DDR in the WT but not in the AC3-I model. (E and F) Simulated central SAN (top) and right atrial (bottom) action potentials from WT (E) salineand (F) Ang II-treated models. The decrease in functional SAN tissue mass due to Ang II infusion slows spontaneous SAN firing rate and RA activation rate. Episodes of transient SAN exit block are observed in the WT Ang II-treated model as evidenced by a SAN action potential (red asterisk) that fails to propagate into the RA free wall region, resulting in an activation pause (red arrow). $V_{m}$, cell membrane potential.

SAN cell apoptosis supports the concept that excessive CaMKII activation by ROS promotes SND by increasing SAN cell death.

We hypothesized that excessive SAN cell death contributed to SND because normal SAN function requires a critical mass of SAN cells to form impulses and electrically capture surrounding atrial tissue. To explore this idea further, we developed a 2-dimensional computational model of the intact SAN based on histologically reconstructed right atrial geometry (Figure 5A and refs. 22, 23). We incorporated measured values for SAN cell loss in WT and AC3-I hearts following saline or Ang II infusion (Figure 5B) to determine whether differences in cell loss observed experimentally (Figure 4) could explain SND in Ang II-treated hearts. We found that increased cell loss observed in WT hearts following Ang II infusion dramatically slowed SAN impulse frequency propagated to the right atrium (Figure 5C), consistent with our experimental findings of slowed HR in Ang II-treated WT mice (Figure 2). The slowed HR in the WT model following Ang II infusion was caused by a decrease in the diastolic SAN depolarization rate (Figure 5D) resulting from increased electrotonic loading of surviving SAN cells by surrounding atrial and non-excitable (e.g., block zone) tissue. In contrast, the AC3-I model, with no differences in cell loss between saline- and Ang II-treated groups, showed no change in right atrial activation or SAN cell diastolic depolarization rate (Figure 5, C and D). Interestingly, while the WT model showed regular right atrial activation following saline treatment (Figure 5E and Supplemental Video 1), Ang II treatment resulted in persistent sinus exit block toward the atrial septum (Supplemental Video 2) and transient block toward the atrial free wall (Figure 5F), consistent with experimental observations (Figure 6). In order to test the model prediction that SND was explicable by SAN cell loss, even in the absence of defects in surviving SAN cells, we experimentally measured spontaneous automaticity in current-clamped SAN cells isolated from WT mice infused with Ang II or saline for 3 weeks. These isolated SAN cells had similar action potential rates (Supplemental Figure 7, A and B), supporting the findings of our model and suggesting that Ang II-induced SND was primarily due to a loss of SAN cell density. In summary, our mathematical model of the intact SAN predicts that cell loss alone can produce SND characterized by slowed HR (atrial activation rate) and defective impulse propagation in the surrounding atrium (sinus exit block). 
A
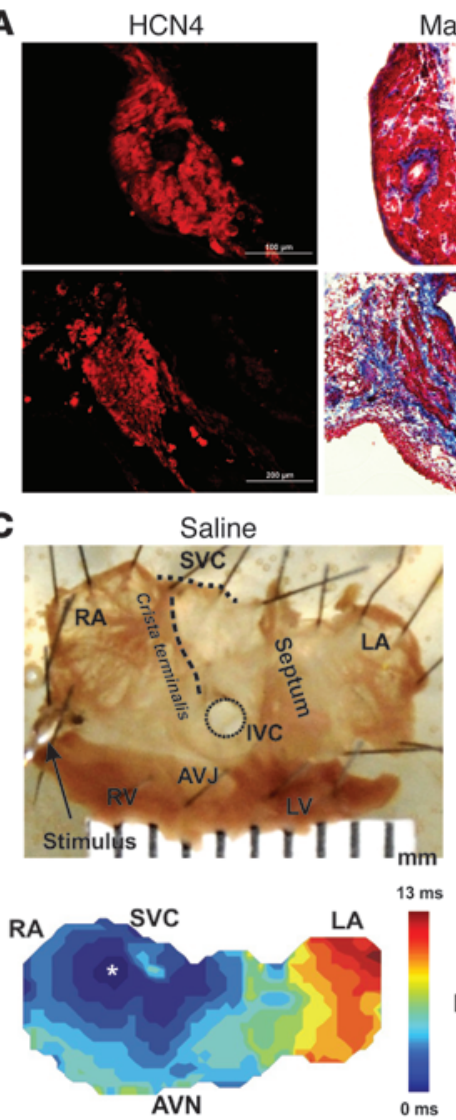

Masson's ' + $80 \%$
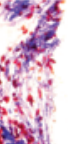

s.

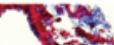

$5+$

2):
B

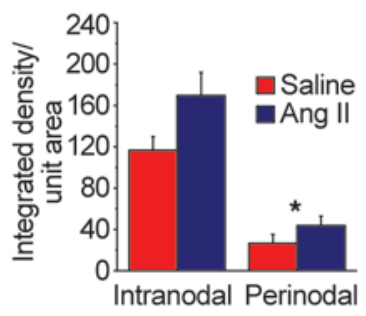

Figure 6

SAN and atrial fibrosis are promoted by Ang II and contribute to SND. (A) Representative samples of Masson's trichrome staining showing increased SAN fibrosis in mice infused with Ang II compared with saline for 3 weeks. Scale bars: $100 \mu \mathrm{m}$. (B) Summary data showing increased fibrosis in the SAN (intranodal, $P=0.06, n=3$ /group) and in SAN-adjacent tissue (perinodal, ${ }^{*} P=0.03, n=3 /$ group). (C) Top row: Representative atrial isolate and conduction velocity measurement. Bottom row: Representative pseudocolored isochrones from optical mapping show decreased conduction velocity (CV) in WT mice infused with Ang II for 3 weeks (lower right panel) compared with WT mice infused with saline for 3 weeks (lower left panel). AVJ, atrioventricular junction; AVN, atrioventricular node; IVC, inferior vena cava; LA, left atrium; LAA, left atrial appendage; RAA, right atrial appendage; SVC, superior vena cava. White asterisks denote the earliest activation in the SAN. (D) Summary data for CV measured from Langendorff-perfused hearts isolated from mice infused with Ang II or saline for 3 weeks. CV was measured in the RA ( $n=5 /$ group, $P=0.07$ baseline and ${ }^{*} P=0.03$ Iso) and LA ( $\#=0.001$ baseline and ${ }^{\dagger} P=0.001$ Iso, $n=5$ /group). The $P$ values represent comparisons between Ang II- and saline-infused mice.

CaMKII inhibition protects against Ang II-induced fibrosis. SAN tissue from patients with SND is marked by increased fibrosis and destruction of normal tissue architecture that are thought to slow conduction and promote defective impulse transmission from the SAN to atrial myocardium $(24,25)$. In order to test whether Ang IIinduced fibrosis is a consequence of CaMKII activation, we quantified collagen with Masson's trichrome staining in the SAN and right atrium of Ang II- and saline-infused mice. Collagen expression within $(P=0.06)$ and bordering $(P=0.03)$ the SAN was higher in WT mice infused with Ang II compared with saline-infused mice (Figure 6, A and B). In contrast, AC3-I mice did not exhibit an increase in fibrosis after 3 weeks of Ang II infusion (Supplemental Figure 8, A and B). These findings show that CaMKII contributes to Ang II-induced SAN and right atrial apoptosis and fibrosis.

Recent investigations have demonstrated that SND involves the "distributed SAN," an area including the SAN and adjacent right atrial myocardium. SND patients exhibit widespread right and left atrial electrophysiological abnormalities, including slowed conduction (7). To determine whether Ang II infusion causes con- duction abnormalities in mice, we measured conduction velocity by optical mapping in the SAN and atria of mice treated with saline or Ang II for 3 weeks. At baseline, Ang II-treated mice had significantly slowed left atrial conduction velocity $(P=0.001)$ and a trend toward slower conduction velocity in right atria $(P=0.07)$ compared with the saline-treated mice (Figure 6, C and D). In the presence of isoproterenol $(3 \mathrm{nM})$, right $(P=0.03)$ and left atria $(P=0.001)$ from Ang II-treated mice had significant conduction slowing compared with saline-infused mice. Thus, histological, biochemical, biophysical, and computational data are all consistent with the concept that chronic exposure to Ang II contributes to SND by reducing SAN cell survival and increasing fibrosis.

Ang II increases ox-CaMKII and causes SND by activating NADPH oxidase. Ang II triggers ROS production that increases ox-CaMKII and apoptosis in ventricular cardiomyocytes by activating NADPH oxidase (11), but to our knowledge NADPH oxidase activity has not been linked to SND. We measured ROS levels in the right atrium of Ang II- and saline-infused WT mice using the ROS indicator dihydroethidium (DHE). ROS levels were signifi- 
A

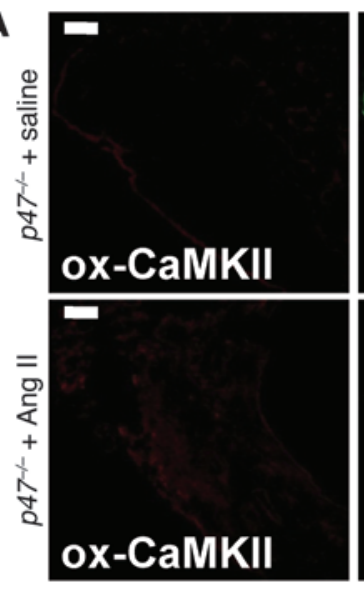

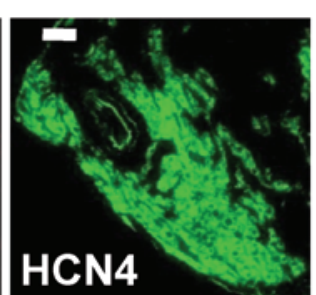

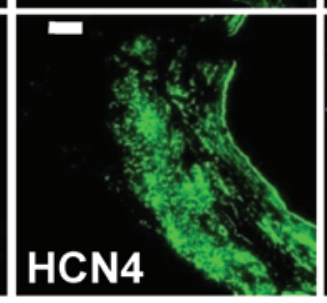

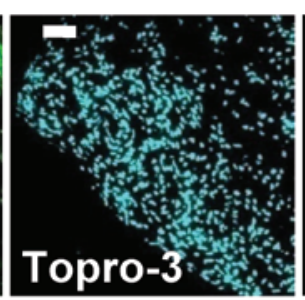
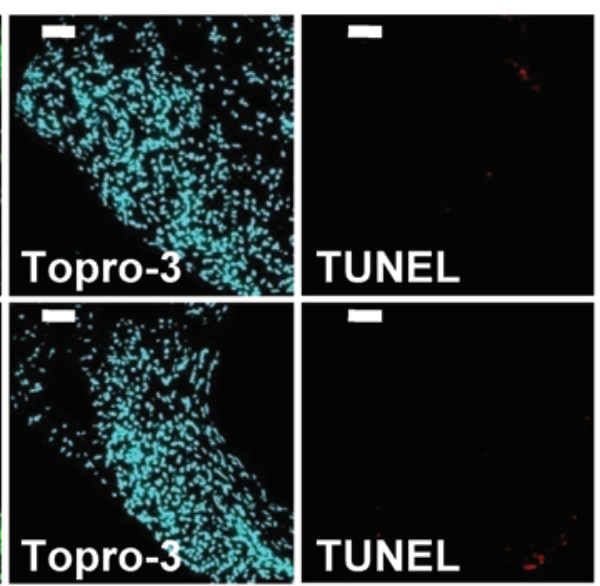

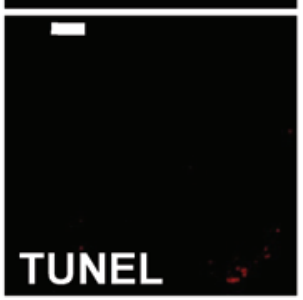

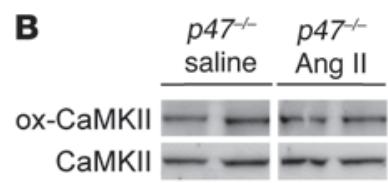

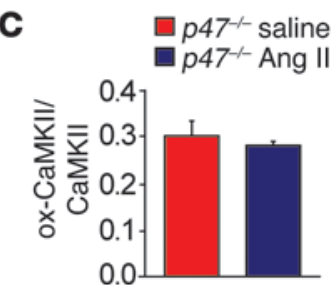

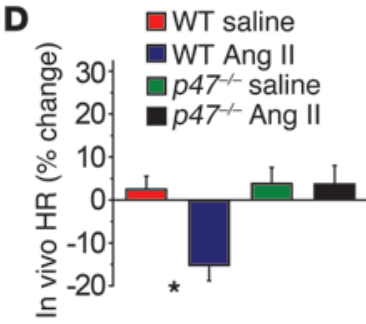
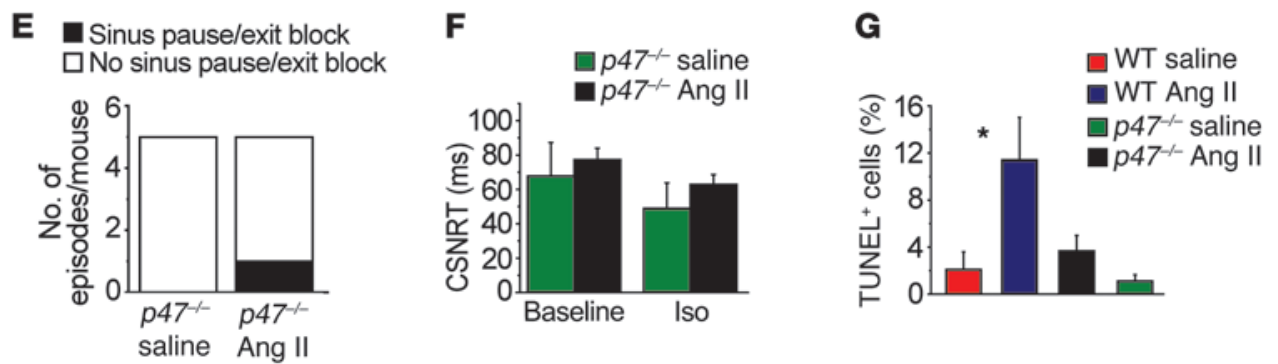

\section{Figure 7}

NADPH oxidase mediates Ang II-induced CaMKII oxidation, apoptosis, and SND. Ang II infusion for 3 weeks in $p 47^{-/-}$mice does not increase ox-CaMKII by immunofluorescence $(\mathbf{A})$ or immunoblotting (B and $\mathbf{C})$. $P=0.54, n=5 /$ group. The lanes were run on the same gel but were noncontiguous. Scale bars: $50 \mu \mathrm{m}$. (D) Unanesthetized and unrestrained $p 47^{-/-}$mice have no significant decrease in resting HR $(P=0.97$, $n=15$ /group) after Ang II compared with saline infusion for 3 weeks. ${ }^{*} P<0.01$ between groups. (E) Langendorff-perfused hearts explanted from mice in $\mathbf{D}$ show no increase in sinus pauses $\left(n=5 /\right.$ group). (F) Ang II infusion for 3 weeks in $p 47^{-/-}$mice does not increase CSNRT $(P=0.66$ for $100 \mathrm{~ms}$ and $P=0.40$ for $80 \mathrm{~ms} ; n=5 /$ group) (G) Summary data showing no increase in TUNEL-positive SAN cells in Ang II- compared with saline-infused $p 47^{-/-}$mice $(P=0.12, n=5 /$ group $)$. ${ }^{*} P=0.01$ between groups.

cantly $(P<0.01)$ elevated in Ang II-treated mice compared with saline-treated controls (Supplemental Figure 9). We next studied $p 47^{-1-}$ mice, which lack a critical subunit of a common myocardial NADPH oxidase isoform (Nox2) (26), to determine whether Ang II contributes to SND by activating NADPH oxidase to increase SAN ox-CaMKII. In contrast to the results in WT mice (Figure 1), ox-CaMKII levels were not elevated in SAN from Ang II-infused compared with saline-infused $p 47^{-1-}$ mice (Figure $\left.7, A-C\right)$. In contrast to Ang II-treated WT mice, $p 47^{-1-}$ mice had no $(P=1)$ reduction in resting HR after 3 weeks of Ang II infusion (Figure 7D). Ang II increased BP in $p 47^{-/-}$mice, similar to WT controls (Supplemental Figure 5), indicating that SND resistance in $p 47^{-1-}$ mice was independent of the hypertensive actions of Ang II, mirroring the findings in AC3-I mice. We next measured HR and CSNRT in Langendorff-perfused hearts isolated from $p 47^{-1-}$ mice previously infused with Ang II or saline for 3 weeks. The $p 47^{-1-}$ mice were resistant to Ang II-mediated SND, as assessed by the number of hearts exhibiting spontaneous pauses (Figure 7E) or by the lack of effect of Ang II infusion on CSNRT (Figure 7F). Ang II-treated $p 47^{-1-}$ mice had a significantly lower frequency of TUNEL-staining SAN cells compared with Ang II-infused WT mice, similar to saline-treated $p 47^{-1-}$ and WT mice (Figure $7 \mathrm{G}$ ). We interpret these data to indicate that NADPH oxidase is required for Ang II-induced ox-CaMKII, increased SAN cell death, and SND.

\section{Discussion}

It has been known for many years that $\mathrm{Ca}^{2+}$-based signaling pathways form the framework for myocardial contraction (27). More recently it has become clear that SAN pacemaker cells also utilize $\mathrm{Ca}^{2+}$ to support physiological increases in HR (3). CaMKII has emerged as a core signaling molecule in contracting and pacemaking myocardial cells, because CaMKII catalyzes the phosphorylation of most major $\mathrm{Ca}^{2+}$ homeostatic proteins (14) that orchestrate the mechanical force-frequency relationship (28) and enable fightor-flight increases in HR (4). Under disease conditions, excessive autonomous CaMKII activity can result from oxidation (29), and a growing body of evidence supports the concept that unconstrained and excessive CaMKII activity contributes to heart failure $(12,30)$, arrhythmias $(12,30-34)$, and sudden death $(35,36)$. CaMKII stimulation of apoptosis is an important component of the cardiomyopathic effects of excessive CaMKII activity $(11,37,38)$. CaMKII inhibition by transgenic expression of inhibitory peptides $(11,12$, $38,39)$, gene deletion $(40,41)$, or drugs $(12,42)$ is protective against common causes of cardiomyopathy, including myocardial infarction and Ang II infusion (11). However, CaMKII has not previously been considered as a disease signal in SND. Our findings show that ox-CaMKII is a marker of human and canine SND and that CaMKII promotes SND by enhancing SAN apoptosis, while CaMKII inhibition can protect against SAN apoptosis and SND in mice. Our 
mouse model of SND involved Ang II infusion, but to our knowledge, there are no reports showing whether angiotensin-converting enzyme inhibitors or angiotensin receptor "blockers" prevent SND in heart failure patients. It will be interesting to learn whether these drugs can reduce or prevent development of SND.

Patients with SND due to rare genetic syndromes may have defects in the HCN4 "pacemaker channel" $(43,44)$ or ankyrins, adapter proteins that localize $\mathrm{Ca}^{2+}$ homeostatic proteins in SAN cells (45). While these genetic forms of SND provide important insights into the molecular mechanisms underlying rare causes of SND, including the potential involvement of ion channel and $\mathrm{Ca}^{2+}$-based signaling pathways, very little is known about the molecular pathways leading to common forms of acquired SND. Here we show that ox-CaMKII may contribute to SND in patients with structural heart disease. In mice, SND can be initiated by activation of NADPH oxidase and culminate in excessive SAN cell death. Accumulating data suggest that chronic heart failure is a condition of augmented oxidative stress (10), but lack of knowledge about defined molecular pathways that determine pathological responses to ROS in heart is a major obstacle in understanding disease mechanisms and developing improved therapies (46). Recently, we reported that ROS activates CaMKII (11), suggesting that ox-CaMKII may contribute to molecular pathways engaged by ROS that are important for common forms of myocardial disease (29). Our current findings suggest that ox-CaMKII is a novel SND biomarker and extend current concepts regarding the role of CaMKII in cardiac pathophysiology by showing that excessive ox-CaMKII causes SND, a known and potentially underappreciated marker of sudden cardiac death (47). Taken together with previous studies (4, 12 ), our new data support the concept that CaMKII inhibition reduces the dynamic range of physiological fight-or-flight responses, but also protects against SND. It will be interesting to learn whether ox-CaMKII is also a factor in other SND models and patient cohorts, to better determine the general applicability of our findings.

Mammalian SAN requires a critical size to adapt its beating rate to the mass of surrounding atrial tissue and to maintain a source/ sink ratio necessary for pacing contractile myocardium. Experience from work with transplanted cellular pacemakers suggests that a critical mass of approximately 300,000 synchronously depolarizing pacemaker cells is required to maintain a stable rhythm, when the cells were implanted into LV myocardium of adult dogs in complete heart block (48). Our study suggests that a critical SAN cell mass is also essential for normal pacemaking and that SND from Ang II infusion is caused by reduction in viable SAN cells. Age-related SND is well recognized (8), but the basis for progressive, age-related reductions in maximal fight-or-flight $\mathrm{HR}$ responses to exercise in healthy persons and animals is not well understood. Old rats have increased myocardial oxidation (49) and reduced HR compared with younger animals, and old rats were recently reported to have a reduced SAN cell mass compared with young rats (17), suggesting the hypothesis that SND due to normal aging could also be due to increased oxCaMKII and SAN cell loss. Our computational model suggests that a critical mass of SAN cells is necessary to maintain a physiological diastolic depolarization rate, impulse formation, and conduction velocity. Selective ablation of HCN4-expressing SAN cells in mice resulted in sick sinus syndrome in a recent study (50), supporting our finding that SAN cell death beyond a critical threshold is sufficient to induce SND. While our experimental and computational data show that CaMKII-triggered SAN cell death is an important determinant of SND, we recognize that CaMKII couples to diverse downstream targets and cellular processes, and our findings do not exclude the possibility that CaMKII may also promote SND through additional pathways.

A large portion of mortality in hospitalized HF patients ( $40 \%)$ may be secondary to $\operatorname{SND}(5,47,51)$. Recent results show that SND is present in $6 \%$ patients with heart failure and is more ominous than ventricular tachycardia as a predictor of death (52). However, at present there are no biological therapeutics to arrest or prevent SND in high-risk patients. Gene therapy with adenoassociated viral vectors encoding the sarcoplasmic endoplasmic reticulum ATPase (SERCA2a) has recently been used for patients with HF (53), suggesting that patients at high risk for SND, but before the onset of SND, undergoing open heart surgery could be treated by SAN painting with viral vectors expressing CaMKIIN to prevent or reduce SND. Our studies in mice provide insights into disease mechanisms underlying common forms of SND and suggest that CaMKII inhibition could be a basis for preventive therapy for patients at high risk for SND.

\section{Methods}

Human samples. The study was approved by the local ethics committee of the Dresden University of Technology (EK790799), and each patient gave written informed consent. The investigation conformed to the principles outlined in the Declaration of Helsinki. Right atrial appendages were obtained from 10 patients with sinus rhythm and 5 patients with SND (Supplemental Table 1) as published previously (54).

Dog atrial samples. The dog experiments were performed with the approval of the IACUC of UCLA. Heart failure was induced in dogs by pacing the right ventricle at 150 beats/min for 3 days, 200 beats $/ \mathrm{min}$ for 3 days, and then 250 beats/min for 3 weeks to induce CHF as previously published (55). The pacemaker was then turned off to allow an additional 2 weeks of ambulatory monitoring. The dogs were then killed, and the atria and ventricles were harvested. Right atrial samples from 3 dogs with pacing-induced heart failure and SND and 3 control dogs were used in this study.

Mouse models. Mice lacking the $p 47$ gene ( $p 47^{-/-}$mice) were purchased from The Jackson Laboratory and backcrossed into the B6D2 background for more than 6 generations. Mice with transgenic myocardial CaMKII inhibition (AC3-I mice) were generated by us, as previously described (12). All experiments were performed in mice $8-12$ weeks of age. The present animal studies were reviewed and approved by the IACUC of the University of Iowa.

$H R$ and BP measurements. One week prior to the start of each experiment, mice were trained on tail cuff BP equipment (BP-2000 Blood Pressure Analysis System, Visitech Systems Inc.). HR and BP recorded on 2 days were averaged as a baseline value. To test the effect of Ang II or saline on HR and $\mathrm{BP}$, mice were followed for 3 weeks. After pump implantation, BP and HR were measured again in the third week and the data averaged.

Mini-osmotic pump implantation. Micro-osmotic pumps (Alzet model 1004, $0.11 \mu \mathrm{l} / \mathrm{h}, 28$ days), containing saline or Ang II ( $3 \mathrm{mg} / \mathrm{kg} / \mathrm{d})$, were inserted subcutaneously under anesthesia (ketamine/xylazine, $87.5 / 12.5 \mathrm{mg} / \mathrm{kg}$ ), as reported previously (11)

ECG and activity telemetry. All telemetry placements were performed 1 week prior to the start of each experiment to allow resolution of the immediate post-surgical HR changes. Mice were anesthetized with ketamine/ xylazine $(87.5 / 12.5 \mathrm{mg} / \mathrm{kg})$ before placement of a transmitter (Data Sciences International) into the abdominal cavity with subcutaneous electrodes in a lead I configuration. Baseline measurements were recorded for 1 week. After insertion of the osmotic minipumps, continuous 24-hour 
recordings were made on alternate days for the 3 -week period. At the end of each experiment, the data were extracted and analyzed for basal HR, HR response to activity, and arrhythmias. We defined spontaneous SND episodes as events of severe bradycardia less than 200 beats/min when the activity level was at 1 or greater.

Transthoracic echocardiography. We recorded transthoracic echocardiograms in conscious mice 3 weeks after Ang II pump implantation, as previously described (56). Images were acquired and analyzed by an operator blinded to mouse genotype and treatment.

Langendorff perfusion and ex vivo ECG recording. ECG recordings from Langendorff-perfused hearts were performed as described previously (4). Excised hearts were quickly mounted on a modified Langendorff apparatus (HSE-HA perfusion systems, Harvard Apparatus) for retrograde aortic perfusion at a constant pressure of $80 \mathrm{mmHg}$ with oxygenated $\left(95 \% \mathrm{O}_{2}\right.$, $5 \% \mathrm{CO}_{2}$ ) Krebs-Henseleit solution consisting of (in mM): $25 \mathrm{NaHCO}_{3}, 118$ $\mathrm{NaCl}, 4.7 \mathrm{KCl}, 1.2 \mathrm{MgSO}_{4}, 1.2 \mathrm{NaH}_{2} \mathrm{PO}_{4}, 2.5 \mathrm{CaCl}_{2}, 0.5 \mathrm{Na}$-EDTA, and 15 glucose, with $\mathrm{pH}$ equilibrated to 7.4 . The perfused hearts were immersed in the water-jacketed bath and were maintained at $36^{\circ} \mathrm{C}$. ECGs were continuously recorded with $\mathrm{Ag}^{+} / \mathrm{AgCl}$ electrodes, which were positioned around the hearts in an approximate Einthoven configuration. We defined SND in Langendorff-perfused hearts as spontaneous pauses when the P-P interval equaled or exceeded twice baseline sinus cycle length.

SNRT/CSNRT measurements. Langendorff-perfused hearts were stimulated with a pair of electrodes placed on the right atrium. Sinus node recovery time (SNRT) was measured after a 30 -second pacing train with a basic cycle length of $100 \mathrm{~ms}$ and $80 \mathrm{~ms}$. For these studies the SNRT was defined as the interval between the last stimulus in the pacing train and the onset of first sinus return beat. The corrected sinus node recovery time (CSNRT) was calculated by subtracting the baseline cycle length from the SNRT.

SAN tissue isolation. Mice were injected with heparin (1,000 IU/ml, i.p.) and avertin $(20 \mu \mathrm{l} / \mathrm{g}$, i.p.). After loss of withdraw reflex, the hearts were excised and placed into Tyrode's solution $\left(35^{\circ} \mathrm{C}\right)$ consisting of (in $\mathrm{mM}$ ): $140 \mathrm{NaCl}, 5.0$ HEPES, 5.5 glucose, $5.4 \mathrm{KCl}, 1.8 \mathrm{CaCl}_{2}, 1.0 \mathrm{MgCl}_{2}$, with $\mathrm{pH}$ adjusted to 7.4 with $\mathrm{NaOH}$. The $\mathrm{SAN}$ region, delimited by the crista terminalis, atrial septum, and orifice of the superior vena cava, was dissected from the heart.

SAN and atrial optical mapping. Optical mapping of spontaneous pacemaker activity and conduction velocity was performed as described previously (57).

Isolated SAN cell action potential recording. SAN cells were isolated, and spontaneous single cell action potentials were recorded in current clamp mode using the perforated patch configuration at $36^{\circ} \mathrm{C}$, as described previously (4).

Immunofluorescence staining and SAN identification. Mice were sacrificed at the end of Ang II or saline infusion. The hearts were excised and placed in Tyrode's solution. Under a dissection microscope, the right atrium was identified and dissected with right atrial appendage, vena cava, and tricuspid valve. The tissue was cryopreserved and sectioned at $14-\mu \mathrm{m}$ thickness. We used a combination of anti-HCN4 and anti-connexin- 43 antibodies to identify the SAN region by positive and negative selection. For all immunofluorescence staining, the slides were air dried for 5 minutes, fixed in formalin for 30 minutes, and washed in PBS, 3 times, 5 minutes each. The sections were incubated with a blocking buffer ( $3 \%$ gelatin from cold water fish skin, $0.1 \%$ Triton $\mathrm{X}$, and $2 \mathrm{mg} / \mathrm{ml} \mathrm{BSA}$ in PBS) with normal serum. The tissue was incubated overnight in the primary antibody to the protein of interest (rabbit anti-mouse ox-CaMKII and mouse anti-C-terminal CaMKII antibodies were developed by us) with HCN4 antibody (rat anti-human HCN4, Abcam). The next day slides were washed in PBS and the tissue incubated with appropriate secondary antibodies for 1 hour. The slides were then washed and coverslipped with VECTASHIELD (Vector Laboratories) with DAPI mounting medium.
ROS detection. Right atria, acquired as described above, were rinsed in cold Tyrode's and flash frozen in OCT mounting compound. The tissue was then sectioned $(30 \mu \mathrm{m})$ and incubated in DHE $\left(2 \times 10^{-6} \mathrm{M}\right)$ for 30 minutes at $37^{\circ} \mathrm{C}$ as previously described (11). Fluorescence was detected with a laser scanning confocal microscope (Zeiss 510 and 710; excitation at $488 \mathrm{~nm}$ and detection at $585 \mathrm{~nm}$ by using a long-pass filter).

Fibrosis detection and quantification. Masson's trichrome staining was used to assess fibrosis. We defined the SAN by HCN4-positive immunostaining $(4,45,58)$ and extra-SAN right atrial tissue by connexin- 43 -positive immunostaining (23). The images were imported into Image (NIH), and the pixels of blue staining were analyzed in the intra-nodal region (within the core SA node), perinodal region (fibrous rim of the SAN), and the right atrium.

Immunoblotting. SAN samples were obtained as described above. Tissue samples were homogenized in modified RIPA buffer (50 mM HEPES, $\mathrm{pH}$ $7.5,150 \mathrm{mM} \mathrm{NaCl}, 5 \mathrm{mM}$ EDTA, $1 \% \mathrm{v} / \mathrm{v}$ NP-40, and $0.5 \% \mathrm{w} / \mathrm{v}$ deoxycholate), containing a mixture of protease and phosphatase inhibitors. Equal amounts of protein were fractionated on NuPAGE gels (Invitrogen) and transferred onto PVDF membranes (Bio-Rad). After blocking nonspecific binding with $10 \% \mathrm{w} / \mathrm{v}$ nonfat milk powder in TBS-T (50 mM Tris- $\mathrm{HCl}$, $\mathrm{pH} 7.6,150 \mathrm{mM} \mathrm{NaCl}$, and $0.1 \% \mathrm{v} / \mathrm{v}$ Tween-20), blots were incubated in primary antibodies (ox-CaMKII and CaMKII) overnight at $4^{\circ} \mathrm{C}$. Blots were washed in TBS-T and incubated with appropriate HRP-conjugated secondary antibodies. Protein bands were detected using ECL reagent (LumiLight, Roche), and loading was routinely monitored by Coomassie staining of the blots after antibody probing and or actin staining. For quantification, Quantity One software (Bio-Rad) was used.

RNA isolation and quantitative RT-PCR. Total RNA was isolated from mouse tissues and cardiomyocytes using RNeasy RNA Isolation Kit (QIAGEN). A 500-ng aliquot of each RNA sample was used for cDNA synthesis in a 50- $\mu \mathrm{l}$ reaction mix, using oligo dT16 as primer and SuperScript III Reverse Transcriptase (Invitrogen). Taqman and SYBR Green-based quantitative realtime PCR reactions were performed. For quantitative RT-PCR (qRT-PCR), $1 \mu \mathrm{l}$ of reverse transcription reaction was mixed with $10 \mathrm{pmol}$ of each specific primer and 12.5 $\mu$ l SYBR PCR Master Mix (Bio-Rad). The reaction was incubated in an iQ5 model thermocycler (Bio-Rad) for 40 cycles consisting of denaturation at $95^{\circ} \mathrm{C}$ for 10 seconds and annealing/extension at $59.9^{\circ} \mathrm{C}$ for 1 minute. The quality of the PCR product was routinely checked by thermal denaturation curve following the qRT-PCR reactions. The threshold cycle $(\mathrm{Ct})$ was determined by using iCycler software (Bio-Rad), and quantification of relative mRNA levels was performed by the $\triangle \Delta \mathrm{Ct}$ method. The following primers were used for Adrb1 qRT-PCR: For, CCTAGAGGGCAAACCTTGTG; Rev, TGCACAGAGTGAGGTAGAGGAC.

Caspase- 3 activity assay. SAN tissue was homogenized in lysis buffer consisting of (in mM): 50 Tris- $\mathrm{HCl}$ pH 7.5, $100 \mathrm{KCl}, 1$ EDTA, 1 EGTA, 1 DTT, 0.1 PMSF, 0.5 benzamidine, $20 \mathrm{mg} / 1$ leupeptin, 1,000 microcystin, 20 sodium pyrophosphate, $50 \mathrm{NaF}$, and 50 sodium $\beta$-glycerophosphate), and total protein content was determined by the Bradford assay. Caspase-3 activity was determined by EnzChek Caspase-3 Assay Kit (Invitrogen).

EIA assay. EIA assay for Ang II was performed on whole blood from saline- and Ang II-infused mice after collection by cardiac puncture in EDTA-coated syringes and mixed with 1:10 0.5 M EDTA and 0.6 trypsin inhibitory units (TIU)/ml aprotinin. Plasma was separated by centrifugation at $1,500 \mathrm{~g}$ for 15 minutes and stored at $-80^{\circ} \mathrm{C}$. The samples were then thawed, and protein was extracted by using Strata solid phase extraction kit according the manufacturer's protocol (SelectScience). Ang II in the individual samples was then quantified using Phoenix Pharmaceuticals enzyme immunoassay kit according to the manufacturer's instructions.

PKA activity. PKA activity was determined by transfer of radioactive $\mathrm{PO}_{4}^{-}$to the PKA substrate Kemptide using a commercial kit according to the suggested protocol (Upstate). SAN tissue samples were isolated and 
immediately frozen in liquid nitrogen. Tissue samples were homogenized in a buffer containing (in mM): 25 Tris, pH 7; 5 EDTA, 10 EGTA; 1 DTT; 1 sodium orthovanadate, and 1 PMSF. Protein kinase activity in each tissue homogenate was determined in a $60-\mu \mathrm{l}$ reaction volume in the presence of $\gamma-{ }^{32} \mathrm{P}-\mathrm{ATP}$ and PKC and CaMKII inhibitors. The specificity of PKA activity was confirmed by addition of the PKA inhibitor peptide PKI. Incorporation of radionuclide into Kemptide was monitored by adsorbing the Kemptide onto P81 phosphocellulose filter paper, followed by scintillation counting. Reactions without the addition of homogenate were used as background controls.

SAN painting. Mice were painted 3-5 days after implantation of Ang II pumps. A poloxamer, trypsin, and collagenase mixture was made with $40 \%$ poloxamer, $1 \%$ trypsin, and $0.25 \%$ collagenase in PBS and then added to equal volume of recombinant adenovirus expressing plasmid for the gene(s) of interest (CaMKIIN-eGFP in an IRES vector or eGFP alone) in solution, making the final concentration of poloxamer, trypsin, and collagenase $20 \%, 0.5 \%$, and $0.125 \%$. The CaMKIIN construct was a gift from Thomas Soderling (Vollum Institute, Portland, Oregon, USA). This mixture is liquid in consistency at $4^{\circ} \mathrm{C}$ but gels at room temperature. Mice were anesthetized using ketamine/xylazine $(87.5 / 12.5 \mathrm{mg} / \mathrm{kg}$, respectively), intubated, and ventilated. Using a right second intercostal muscle incision, the heart was visualized under the dissecting microscope and the gel applied to the posterior surface of the junction of the superior vena cava and right atrium with a fine brush 3 times with intervening periods of drying. The intercostal muscles, pectoralis major and minor, and the skin incision were closed using $6 / 0$ silk, and the mice were allowed to recover.

Mathematical model of the SAN. Model geometry was represented using a 2dimensional $400 \times 45$ node rectangular grid (spatial resolution of $40 \mu \mathrm{m}$ ) and histologically reconstructed sections through the rabbit right atrium (Figure $5 \mathrm{~A}$ ) (merge of sections corresponding to $z$ coordinates $4.6 \mathrm{~mm}$ and $4.7 \mathrm{~mm}$ ) $(22,23)$. Each node was assigned a flag corresponding to a cell type based on immunohistochemistry data. Cells with decreased excitability were introduced between the SAN and atrial septum to reproduce the experimentally observed "block zone" in this region (22). Cell loss in the SAN due to apoptosis was simulated by replacing normal SAN cells selected at random with inexcitable cells, poorly coupled to surrounding tissue. Detailed mathematical models were used to simulate central SAN cell, peripheral SAN cell, and atrial action potentials (59-61). Regional differences in cell size, capacitance, and coupling were taken into account (Supplemental Table 3). The 2-dimensional cable equation describing action potential propagation was solved using an alternating direction implicit method and a time step of $0.005 \mathrm{~ms}(62)$.

Statistics. Data are presented as mean \pm SEM. $P$ values were assessed with Student's $t$ test (2-tailed) or ANOVA, as appropriate, for continuous data. The Tukey's test was used for post hoc testing. Discrete variables were analyzed by Fisher's exact test. The null hypothesis was rejected for $P$ values less than 0.05 .

\section{Acknowledgments}

Kathy Zimmerman performed mouse echocardiograms. This work was funded by NIH grants R01 HL 079031, R01 HL 096652, R01 HL 070250, R01 HL 071140, and RR026293; the European Union (European Network for Translational Research in Atrial Fibrillation FP7 grant); the University of Iowa Research Foundation; two Fondation Leducq grants: "Transatlantic Alliance for CaMKII Signaling” (08CVD01 to M.E. Anderson, J.K. Donahue, P.J. Mohler, and T.J. Hund) and "European-North American Atrial Fibrillation Research Alliance” (07CVD03 to D. Dobrev). P.D. Swaminathan's salary was supported by a Kenneth M. Rosen Fellowship and Max Schaldach Fellowship from the Heart Rhythm Society and a University of Iowa Cardiovascular Center Interdisciplinary Research Fellowship. A. Purohit's salary was supported by AHA postdoctoral fellowship grant 10POST3620047. T.J. Hund has support from NIH grant R00 HL 096805 and the Gilead Sciences Research Scholars Program in Cardiovascular Disease. We thank Maria Scheel and the University of Iowa Gene Transfer Vector Core, an NIH-funded resource, for generating virus for these studies and Shawn Roach for graphic art. The authors thank Michael Knaut and Klaus Matschke of Heart Centre Dresden for providing human atrial tissue and Annett Opitz for excellent technical assistance.

Received for publication March 3, 2011, and accepted in revised form May 25, 2011.

Address correspondence to: Mark E. Anderson, 285 Newton Road, CBRB 2256, Iowa City, Iowa 52242, USA. Phone: 319.356.2745; Fax: 319.356.8608; E-mail: mark-e-anderson@uiowa.edu. Or to: Thomas J. Hund, Davis Heart and Lung Research Institute, 473 W. 12th Avenue, Columbus, Ohio 43210, USA. Phone: 614.247.7766; Fax: 614.247.7799; E-mail: Thomas.Hund@osumc.edu.
1. DiFrancesco D. Characterization of single pacemaker channels in cardiac sino-atrial node cells Nature. 1986;324(6096):470-473.

2. Vinogradova TM, Zhou YY, Maltsev V, Lyashkov A, Stern M, Lakatta EG. Rhythmic ryanodine receptor $\mathrm{Ca} 2+$ releases during diastolic depolarization of sinoatrial pacemaker cells do not require membrane depolarization. Circ Res. 2004;94(6):802-809.

3. Lakatta EG, Maltsev VA, Vinogradova TM. A coupled SYSTEM of intracellular Ca2+ clocks and surface membrane voltage clocks controls the timekeeping mechanism of the heart's pacemaker. Circ Res. 2010;106(4):659-673.

4. Wu Y, et al. Calmodulin kinase II is required for fight or flight sinoatrial node physiology. Proc Natl Acad Sci U S A. 2009;106(14):5972-5977.

5. Uretsky BF, Sheahan RG. Primary prevention of sudden cardiac death in heart failure: will the solution be shocking? J Am Coll Cardiol. 1997;30(7):1589-1597.

6. Opthof T, Coronel R, Rademaker HM, Vermeulen JT, Wilms-Schopman FJ, Janse MJ. Changes in sinus node function in a rabbit model of heart failure with ventricular arrhythmias and sudden death. Circulation. 2000;101(25):2975-2980.

7. Sanders P, Kistler PM, Morton JB, Spence SJ, Kalman
JM. Remodeling of sinus node function in patients with congestive heart failure: reduction in sinus node reserve. Circulation. 2004;110(8):897-903.

8. Fuster V, O'Rourke RA, Roberts R, King SB, Nash IS, Prystowski EN, eds. Hurst's the Heart. New York, New York, USA: McGraw-Hill Medical Publishing Division; 2004.

9. Mudd JO, Kass DA. Tackling heart failure in the twenty-first century. Nature. 2008;451(7181):919-928.

10. Giordano FJ. Oxygen, oxidative stress, hypoxia, and heart failure. J Clin Invest. 2005;115(3):500-508.

11. Erickson JR, et al. A dynamic pathway for calciumindependent activation of CaMKII by methionine oxidation. Cell. 2008;133(3):462-474.

12. Zhang R, et al. Calmodulin kinase II inhibition protects against structural heart disease. Nat Med. 2005;11(4):409-417.

13. Singh MV, et al. $\mathrm{Ca} 2+/$ calmodulin-dependent kinase II triggers cell membrane injury by inducing complement factor B gene expression in the mouse heart. J Clin Invest. 2009;119(4):986-996.

14. Couchonnal LF, Anderson ME. The role of calmodulin kinase II in myocardial physiology and disease. Physiology (Bethesda). 2008;23:151-159.

15. Swedberg K, Eneroth P, Kjekshus J, Wilhelmsen L.
Hormones regulating cardiovascular function in patients with severe congestive heart failure and their relation to mortality. CONSENSUS Trial Study Group. Circulation. 1990;82(5):1730-1736.

16. Umeda M, Ichikawa S, Kanda $T$, Sumino $H$, Kobayashi I. Hormone replacement therapy increases plasma level of angiotensin II in postmenopausal hypertensive women. Am J Hypertens. 2001; 14(3):206-211.

17. de Melo SR, de Souza RR, Mandarim-de-Lacerda CA. Stereologic study of the sinoatrial node of rats - age related changes. Biogerontology. 2002;3(6):383-390.

18. Gomes JA, Hariman RI, Chowdry IA. New application of direct sinus node recordings in man: assessment of sinus node recovery time. Circulation. 1984;70(4):663-671.

19. Castellano M, Bohm M. The cardiac beta-adrenoceptor-mediated signaling pathway and its alterations in hypertensive heart disease. Hypertension. 1997;29(3):715-722.

20. Chang BH, Mukherji S, Soderling TR. Characterization of a calmodulin kinase II inhibitor protein in brain. Proc Natl Acad Sci U S A. 1998; 95(18):10890-10895. 
21. Kikuchi K, McDonald AD, Sasano T, Donahue JK. Targeted modification of atrial electrophysiology by homogeneous transmural atrial gene transfer. Circulation. 2005;111(3):264-270.

22. Butters TD, et al. Mechanistic links between $\mathrm{Na}^{+}$ channel (SCN5A) mutations and impaired cardiac pacemaking in sick sinus syndrome. Circ Res. 2010;107(1):126-137.

23. Dobrzynski H, et al. Computer three-dimensional reconstruction of the sinoatrial node. Circulation. 2005;111(7):846-854

24. Ferrer MI. The etiology and natural history of sinus node disorders. Arch Intern Med. 1982; 142(2):371-372.

25. Gaffney BJ, Wasserman AG, Rotsztain A, Rios JC. Sick sinus syndrome: mechanisms and management. Cardiovasc Clin. 1980;11(1):7-25.

26. Cave AC, et al. NADPH oxidases in cardiovascular health and disease. Antioxid Redox Signal. 2006; 8(5-6):691-728

27. Fabiato A, Fabiato F. Contractions induced by a calcium-triggered release of calcium from the sarcoplasmic reticulum of single skinned cardiac cells J Physiol. 1975;249(3):469-495.

28. Kushnir A, Shan J, Betzenhauser MJ, Reiken S, Marks AR. Role of CaMKIIdelta phosphorylation of the cardiac ryanodine receptor in the force frequency relationship and heart failure. Proc Natl Acad Sci U S A. 2010;107(22):10274-10279.

29. Erickson JR, He BJ, Grumbach IM, and Anderson ME. CaMKII in the cardiovascular system: sensing redox states. Physiol Rev. In press.

30. Yeh YH, et al. Calcium-handling abnormalities underlying atrial arrhythmogenesis and contractile dysfunction in dogs with congestive heart failure. Circ Arrbythm Electrophysiol. 2008;1(2):93-102

31. Chelu MG, et al. Calmodulin kinase II-mediated sarcoplasmic reticulum Ca2+ leak promotes atrial fibrillation in mice. JClin Invest. 2009;119(7):1940-1951.

32. Wu Y, et al. Calmodulin kinase II and arrhythmias in a mouse model of cardiac hypertrophy. Circulation. 2002;106(10):1288-1293.

33. van Oort RJ, et al. Ryanodine receptor phosphorylation by calcium/calmodulin-dependent protein kinase II promotes life-threatening ventricular arrhythmias in mice with heart failure. Circulation 2010;122(25):2669-2679.

34. Dobrev D, Voigt N, Wehrens XH. The ryanodine receptor channel as a molecular motif in atrial fibrillation: pathophysiological and therapeutic implications. Cardiovasc Res. 2011;89(4):734-743.

35. Khoo MS, et al. Death, cardiac dysfunction, and arrhythmias are increased by calmodulin kinase II in calcineurin cardiomyopathy. Circulation. 2006;114(13):1352-1359.

36. Zhang $\mathrm{T}$, et al. The deltaC isoform of CaMKII is activated in cardiac hypertrophy and induces dilated cardiomyopathy and heart failure. Circ Res. 2003;92(8):912-919.

37. Yang Y, et al. Calmodulin kinase II inhibition protects against myocardial cell apoptosis in vivo. Am J Physiol Heart Circ Physiol. 2006;291(6):H3065-H3075.

38. Palomeque J, et al. Angiotensin II-induced oxidative stress resets the $\mathrm{Ca} 2+$ dependence of $\mathrm{Ca} 2+$ calmodulin protein kinase II and promotes a death pathway conserved across different species. Circ Res. 2009;105(12):1204-1212.

39. Toko $\mathrm{H}$, et al. Ca2+/calmodulin-dependent kinase IIdelta causes heart failure by accumulation of p53 in dilated cardiomyopathy. Circulation. 2010;122(9):891-899.

40. Ling $\mathrm{H}$, et al. Requirement for $\mathrm{Ca} 2+/$ calmodulin-dependent kinase II in the transition from pressure overload-induced cardiac hypertrophy to heart failure in mice. J Clin Invest. 2009; 119(5):1230-1240.

41. Backs J, et al. The delta isoform of CaM kinase II is required for pathological cardiac hypertrophy and remodeling after pressure overload. Proc Natl Acad Sci U S A. 2009;106(7):2342-2347.

42. Liu N, et al. Calmodulin kinase II inhibition prevents arrhythmias in RyR2(R4496C+/-) mice with catecholaminergic polymorphic ventricular tachycardia. J Mol Cell Cardiol. 2011;50(1):214-222.

43. Schulze-Bahr E, et al. Pacemaker channel dysfunction in a patient with sinus node disease. $J$ Clin Invest. 2003;111(10):1537-1545.

44. Milanesi R, Baruscotti M, Gnecchi-Ruscone T, DiFrancesco D. Familial sinus bradycardia associated with a mutation in the cardiac pacemaker channel. NEngl J Med. 2006;354(2):151-157.

45. Le Scouarnec S, et al. Dysfunction in ankyrin-Bdependent ion channel and transporter targeting causes human sinus node disease. Proc Natl Acad Sci US A. 2008;105(40):15617-15622.

46. Tomaselli GF, Barth AS. Sudden cardio arrest: oxidative stress irritates the heart. Nat Med. 2010; 16(6):648-649.

47. Stevenson WG, Stevenson LW, Middlekauff HR, Saxon LA. Sudden death prevention in patients with advanced ventricular dysfunction. Circulation. 1993;88(6):2953-2961.

48. Plotnikov AN, et al. Xenografted adult human mesenchymal stem cells provide a platform for sustained biological pacemaker function in canine heart. Circulation. 2007;116(7):706-713.

49. Maurel A, et al. Age-dependent increase in hydrogen peroxide production by cardiac monoamine oxidase A in rats. Am J Physiol Heart Circ Physiol. 2003;284(4):H1460-H1467.

50. Herrmann S, Fabritz L, Layh B, Kirchhof P, Ludwig A. Insights into sick sinus syndrome from an induc- ble mouse model. Cardiovasc Res. 2011;90(1):38-48. 51. Faggiano P, d'Aloia A, Gualeni A, Gardini A, Giordano A. Mechanisms and immediate outcome of in-hospital cardiac arrest in patients with advanced heart failure secondary to ischemic or idiopathic dilated cardiomyopathy. Am J Cardiol. 2001; 87(5):655-657.

52. Bloch Thomsen PE, et al. Long-term recording of cardiac arrhythmias with an implantable cardiac monitor in patients with reduced ejection fraction after acute myocardial infarction: the cardiac arrhythmias and risk stratification after acute myocardial infarction (CARISMA) study. Circulation. 2010;122(13):1258-1264.

53. Jaski BE, et al. Calcium upregulation by percutaneous administration of gene therapy in cardiac disease (CUPID Trial), a first-in-human phase $1 / 2$ clinical trial. J Card Fail. 2009;15(3):171-181.

54 . Voigt $\mathrm{N}$, et al. Left-to-right atrial inward rectifier potassium current gradients in patients with paroxysmal versus chronic atrial fibrillation. Circ Arrbythm Electrophysiol. 2010;3(5):472-480.

55. Ogawa $\mathrm{M}$, et al. Left stellate ganglion and vagal nerve activity and cardiac arrhythmias in ambulatory dogs with pacing-induced congestive heart failure. J Am Coll Cardiol. 2007;50(4):335-343.

56. Weiss RM, Ohashi M, Miller JD, Young SG, Heistad DD. Calcific aortic valve stenosis in old hypercholesterolemic mice. Circulation. 2006; 114(19):2065-2069.

57. Glukhov AV, Fedorov VV, Anderson ME, Mohler PJ, Efimov IR. Functional anatomy of the murine sinus node: high-resolution optical mapping of ankyrin-B heterozygous mice. Am J Physiol Heart Circ Physiol. 2010;299(2):H482-H491.

58. Liu J, Dobrzynski H, Yanni J, Boyett MR, Lei M. Organisation of the mouse sinoatrial node: structure and expression of $\mathrm{HCN}$ channels. Cardiovasc Res. 2007;73(4):729-738.

59. Kurata Y, Hisatome I, Imanishi S, Shibamoto T. Dynamical description of sinoatrial node pacemaking: improved mathematical model for primary pacemaker cell. Am J Physiol Heart Circ Physiol. 2002;283(5):H2074-H2101.

60. Kurata Y, Matsuda H, Hisatome I, Shibamoto T. Regional difference in dynamical property of sinoatrial node pacemaking: role of na+ channel current. Biophys J. 2008;95(2):951-977.

61. Courtemanche M, Ramirez RJ, Nattel S. Ionic mechanisms underlying human atrial action potential properties: insights from a mathematical model. Am J Physiol. 1998;275(1 pt 2):H301-H321.

62. Joyner RW, Ramon F, Morre JW. Simulation of action potential propagation in an inhomogeneous sheet of coupled excitable cells. Circ Res. 1975;36(5):654-661. 